\title{
Sliding-Mode Controller for Bilateral Teleoperation with Varying Time Delay
}

\author{
Jong Hyeon Park and Hyun Chul Cho \\ School of Mechanical Engineering \\ Hanyang University \\ Seoul, 133-791, Korea \\ email:jongpark@email.hanyang.ac.kr
}

\begin{abstract}
Bilateral teleoperation systems, connected to computer networks such as Internet have to deal with varying communication time delay. And the entire system is easy to become unstable due to irregular time delay. In this paper, we design a sliding-mode controller for the slave and an impedance controller for the master. We propose a modifled sliding-mode controller, in which the nonlinear gain can be set independently of the time delay variation. The proposed controller effectively compensates the effects of the varying time delay which deteriorates the performance of the regular sliding-mode controller. We illustrate the validity of the proposed controller using simulations with 1-DOF master/slave bilateral teleoperation system.
\end{abstract}

Keywords - Sliding-Mode Control, Bilateral Teleoperation, Varying Time Delay.

\section{INTRODUCTION}

Teleoperation enables a operator to manipulate remote objects. One of the main goals in teleoperation researches is to provide the operator with the feeling of telepresence, i.e., being present at the remote site. To provide good telepresence sophisticated control methods are in demand. Teleoperation systems having force-feedback ability can considerably improve the overall system performance in this context [12].

It is difficult, however, to control such a bilateral teleoperation system when communication time delays between its master and slave exist. Anderson and Spong [1] pointed out that even a small constant time delay can make bilateral systems unstable and certainly degrade the operator's intuition and performance. In traditional communication ways for a bilateral teleoperation, there are a communication satellite, a private communication cable, a private radio channel and so on. And these ways can be assumed to make the communication block have a constant time delay between its input and output ends [7].

Recently, many people use a computer network as the communication block of a bilateral teleoperation. The teleoperation systems using computer networks such as Internet are easy to make and have high flexibility compared to the conventional systems using private communication channels. But, in this case, the problem lies in the fact that communication time delay between a master and a slave varies with direction according to the condition of the network. Because of this varying time delay, communication block using the computer network becomes a time-varying system, and several existing control methods [1], [3], [8], [9] cannot be applied directly. For example, Anderson and Spong have proposed a method to stabilize a bilateral teleoperation system with a constant time delay using the passivity and scattering operator [1]. However, the passivity of communication block is not stabilized by the scattering transformation when the communication block has a varying time delay [6].

Several control methods have been proposed to overcome such a varying time delay problem. Oboe and Fiorini [2] presented a design environment for the identification, control design, and test of a telerobotic system connected to the Internet. They also proposed a quasi-optimal estimator to compensate small data losses. Brady and Tarn [4] discussed a description of the delays inherent in communication channels and presented a state space model taking into account the time-varying nature of the delay. Besides Sano et al. [5] designed a gain-scheduled $H_{\infty}$ controller to compensate a varying time delay. Kikuchi et al. [6] used the virtual time delay method to keep the apparent time delay constant. In addition to this, they used the environment predictive display system to estimate the behavior of the environment and to give the predicted behavior to the operator. The virtual time delay method was also used in Kosuge and Murayama [7]. Finally, Niemeyer and Slotine [10] showed that stability of bilateral teleoperation in the presence of irregular time delay can be preserved through the systematic use of wave-variable filters.

In this paper, we propose an alternative sliding-mode 
controller for a bilateral teleoperation with a varying time delay. In a regular sliding-mode controller, the sliding condition of the controlled system is implicitly a function of a time delay and external forces. Therefore, in order to satisfy the sliding condition with the regular sliding-mode controller, the nonlinear gain must be tuned according to a measured time delay. However, it is not so easy to measure the time delay between the master and slave under implementation. Moreover, as the time delay increases, the nonlinear gain becomes larger, which leads an actuator saturation problem and/or an input chattering. It is shown that some modification to the regular sliding-mode controller enables the entire slave system to perform the task well independently of time delay.

Section 2 describes the dynamics of the bilateral teleoperation system and discusses some communication protocol issues in applying for a manipulator control. Section 3 derives the regular sliding-mode controller and the proposed one, which performs well even in the presence of an irregular time delay, with the impedance controller. Simulation results of the proposed controller are shown in Section 4, followed by conclusions in Section 5.

\section{Bilateral Teleoperation System}

A master device is manipulated by its human operator and a slave manipulator executes real tasks in a remote site. For the bilateral teleoperation, the velocity and force of the master are transmitted to the slave and the force and the velocity information of the slave is sent to the master by a communication protocol such as TCP, UDP, and ICMP.

In this paper, a 1-DOF master/slave system is considered. The dynamics of the master and slave are expressed as a 1-DOF mass-damper system and the schematic diagram of the model is shown in Fig. 1.

$$
\begin{aligned}
M_{m} \dot{v}_{m}+B_{m} v_{m} & =u_{m}+f_{h} \\
M_{s} \dot{v}_{s}+B_{s} v_{s} & =u_{s}-f_{e}
\end{aligned}
$$

where $v$ and $u$ are velocities and torques; $M$ and $B$ are masses and viscous coefficients; subscript ' $m$ ' and ' $s$ ' denote the master and slave, respectively. Finally, $f_{h}$ is the force applied by the operator on the master, and $f_{e}$ is the force applied by the slave on the environment.

As mentioned earlier, a computer network such as Internet is used for communication block in this paper. The two main factors influencing Internet-based teleoperation are the transmission time delay between the master and the slave and the loss of data packets owing to network congestion [2]. Before reaching its destination, information on velocity and force traverses

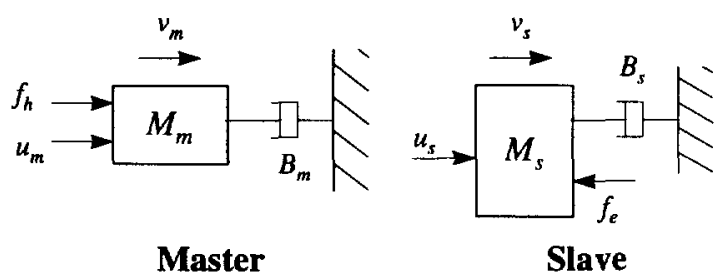

Fig. 1. Dynamic models of the master and slave.

several computers, called nodes. Each node may route them, each time, to different node and/or discard some data packets according to the node condition. This introduces an irregular communication time delay and possibly some data may be even lost on its way.

In the Internet-communication, TCP and UDP are used as the standard communication protocol. Oboe and Fiorini [2] have said that since the TCP protocol introduces an excessive overhead and cannot be excluded by the user, the UDP protocol is preferred for constant data-flow applications, such as control. There is, however, no reordering ability in the UDP protocol. In other words, the orders of the sending data packets may be changed when transmitted with the UDP. This can cause the abrupt changes in data values, which deteriorate the system performance. Under the assumption of no data packet loss, a controller for the bilateral teleoperation is designed. If the TCP is used as an Internet communication protocol, data loss and ordering problems can be neglected owing to the TCP's error recovery and reordering abilities.

\section{iII. Controller Design}

In this section, the impedance control is used for the master device and the sliding-mode control for the slave. First, each controller is designed for the case that there is no time delay, then these controllers are modified in order to apply for the varying time delay case. Finally, the modified sliding-mode controller is derived in consideration of the boundary of the nonlinear gain.

Buttolo et al. [11] have derived various forms of the sliding control law for bilateral teleoperation systems with an arbitrary factor for force and position scaling. There is, however, no time delay consideration in the teleoperation, and there are some restriction in applying for Internet-based teleoperation systems.

\section{A. Controller Design for the Master/Slave without Time Delay}

An impedance controller and a sliding-mode controller for the bilateral teleoperation system without time delay are designed. First, using scaling factors, 


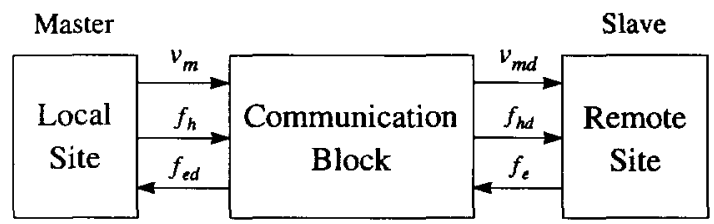

Fig. 2. Block diagram of the bilateral teleoperation.

the relationships between the master and the slave in position and force are defined as:

$$
\begin{aligned}
x_{s} & =k_{p} x_{m d} \\
f_{h} & =k_{f} f_{e d}
\end{aligned}
$$

where $x_{m d}$ and $x_{s}$ are the position command from the master and the slave position, respectively; $f_{e d}$ is the external force command from the slave to the master. Scaling factor $k_{p}$ and $k_{f}$ are constant and are determined by a human operator according to the characteristics of his or her task.

In the bilateral teleoperation, the master device must reflect to the human operator the contact force between the slave manipulator and the environment. With the impedance control, an impact force imposed on a human can be reduced effectively and desired characteristics between the operating force and external force can be determined. For this, a desired impedance characteristic is specified for the master such that

$$
M \ddot{x}_{m}+B \dot{x}_{m}+K x_{m}=f_{h}-k_{f} f_{e}
$$

where $M, B$ and $K$ are the inertia, damping and stiffness matrices of the desired impedance, respectively.

If the measurement of the operator force, $f_{h}$ can be measured, the control law to achieve the impedance model of eq. (5) can be obtained. By combining eq. (5) with (1) to remove acceleration $\ddot{x}_{m}$, the control input to the master can be represented as

$$
\begin{aligned}
u_{m}= & \left(B_{m}-\frac{M_{m}}{M} B\right) v_{m}+\left(\frac{M_{m}}{M}-1\right) f_{h} \\
& -\frac{M_{m}}{M}\left(k_{f} f_{e}+K x_{m}\right)
\end{aligned}
$$

Note that $f_{h}$ could have been removed instead of $\ddot{x}_{m}$ by using eq. (5) and eq. (1). This would not require the measurement of the operating force $f_{h}$; however, the control law for the master device would include a term of $\ddot{x}_{m}$, which is very difficult to measure precisely due to noises.

Next, a sliding-mode controller is designed for the slave manipulator to track the position of the master.
A sliding-mode controller is known as being robust for model parameter variations. This property is used for compensating the effects of a time delay and its variation later. Let $\tilde{x}(t)=x_{s}(t)-k_{p} x_{m}(t)$ be the tracking error between the positions of the master and the slave, and let us define sliding surface, $s(t)$ as:

$$
s(t)=\dot{\tilde{x}}(t)+\lambda \tilde{x}(t)
$$

where $\lambda$ is a strictly positive constant, and its derivative with respect to the time is:

$$
\dot{s}(t)=\left(\ddot{x}_{s}-k_{p} \ddot{x}_{m}\right)+\lambda\left(\dot{x}_{s}-k_{p} \dot{x}_{m}\right)
$$

For the equivalent control [13], substituting $\ddot{x}_{s}$ which satisfies $\dot{s}=0$ and $\ddot{x}_{m}$ in eq.(5) into eq. (2) gives:

$$
\begin{aligned}
u_{s}= & B_{s} v_{s}+f_{e}-\frac{k_{p} M_{s}}{M}\left(B v_{m}-f_{h}+k_{f} f_{e}+K x_{m}\right) \\
& -M_{s} \lambda \dot{\tilde{x}}
\end{aligned}
$$

When there is no significant modeling uncertainties and no time delay, with these control laws, eq. (6) and eq. (9), the master will reflect properly the contact force of the slave to the operator and the slave will follow the position of the master exactly.

In Buttolo et al. [11], one of the control laws must be selected to involve a negative feedback term proportional to $f_{e}$ in the controller depending on the values of the desired parameters $k_{p}, k_{f}$. But, in eq. (9), this is not the case.

\section{B. Controller Design for the Master/Slave with Vary- ing Time Delay}

A bilateral teleoperation system can be represented by the block diagram of Fig. 2 . In Fig. $2, v_{m d}$ and $f_{h d}$ are the command velocity and the operating force, respectively, transmitted from the master to the slave. Using the computer network as the communication block, the time delay between the master and slave varies with direction and according to the network condition as follows:

$$
\begin{aligned}
v_{m d}(t) & =v_{m}\left(t-T_{1}(t)\right) \\
f_{h d}(t) & =f_{h}\left(t-T_{1}(t)\right) \\
f_{e d}(t) & =f_{e}\left(t-T_{2}(t)\right)
\end{aligned}
$$

where $T_{1}(t)$ is the time delay from the master to the slave, and $T_{2}(t)$ is the time delay from the slave to the master. Note that these delays vary with respect to the time.

With these delayed signals, control laws for the master and slave, eq. (6) and (9), can be rewritten as follows: 


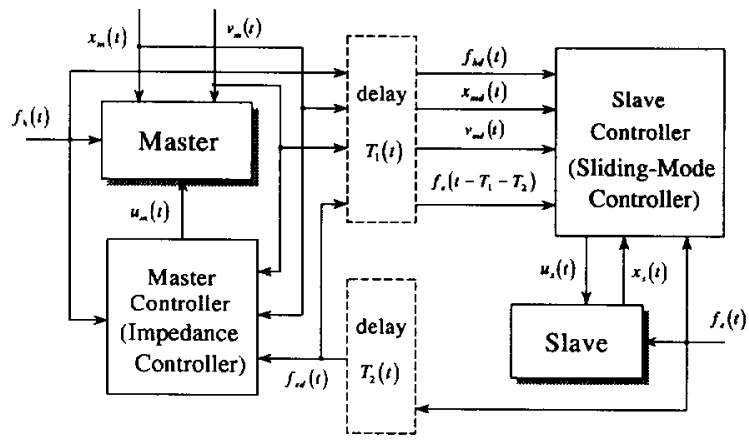

Fig. 3. Overall system block diagram.

$$
\begin{aligned}
u_{m}(t)= & \left(B_{m}-\frac{M_{m}}{M} B\right) v_{m}(t)+\left(\frac{M_{m}}{M}-1\right) f_{h}(t) \\
& -\frac{M_{m}}{M}\left\{k_{f} f_{e d}(t)+K x_{m}(t)\right\} \\
u_{s}(t)= & B_{s} v_{s}(t)+f_{e}(t)-\frac{k_{p} M_{s}}{M}\left\{B v_{m d}(t)-f_{h d}(t)\right. \\
& \left.+k_{f} f_{e}(t)+K x_{m d}(t)\right\} \\
& -M_{s} \lambda \dot{\tilde{x}}_{d}(t)-K_{g a i n} \cdot \operatorname{sat}\left(\frac{s_{d}}{\Phi}\right)
\end{aligned}
$$

where $K_{\text {gain }}$ is the nonlinear gain, sat $(\cdot)$ is a saturation function, and $\Phi$ is the boundary layer thickness, which reduces the chattering of the control input. In the above equation, the new sliding surface, $s_{d}(t)=\dot{\tilde{x}}_{d}(t)+\lambda \tilde{x}_{d}(t)$ with $\tilde{x}_{d}(t)=x_{s}(t)-k_{p} x_{m d}(t)$ is used.

In eq. (14), the sat $(\cdot)$-term is used to compensate the time delay effect. When the transmitted signals are delayed, however, the performance of a regular slidingmode controller would be easily deteriorated by the irregular time delay, and this can be investigated through the following analysis.

Combining eq. (2) with eq. (14) in the expression of $s_{d}$ leads to:

$\dot{s}_{d}(t)+\frac{k_{p} k_{f}}{M}\left\{f_{e}(t)-f_{e}\left(t-T_{1}-T_{2}\right)\right\}+\frac{K_{g a i n}}{M_{s}} \operatorname{sat}\left(\frac{s_{d}}{\Phi}\right)=0$

To satisfy the sliding condition, $\dot{s}_{d} s_{d} \leq-\eta\left|s_{d}\right|, K_{\text {gain }}$ must be:

$$
K_{g a i n} \geq M_{s} \eta+k_{p} k_{f} \frac{M_{s}}{M}\left|f_{e}(t)-f_{e}\left(t-T_{1}-T_{2}\right)\right|
$$

As the round-trip time (RTT) increases, the gain, $K_{\text {gain }}$ must be set to larger value so that the controlled system satisfies the sliding condition. But, larger $K_{\text {gain }}$

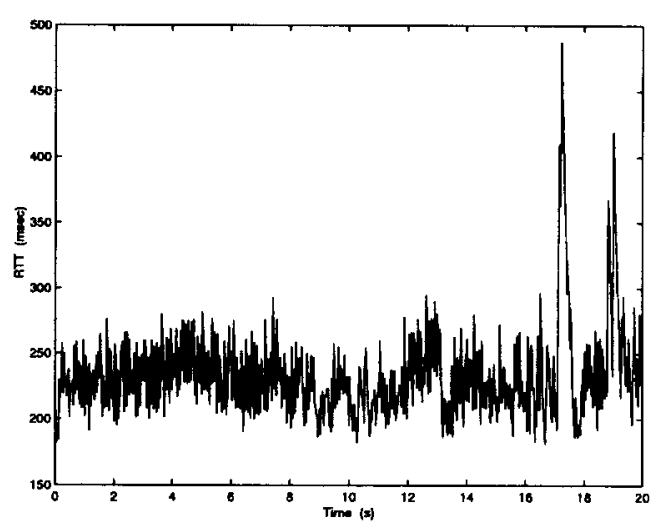

Fig. 4. Time delay from the master to the slave used for the simulation.

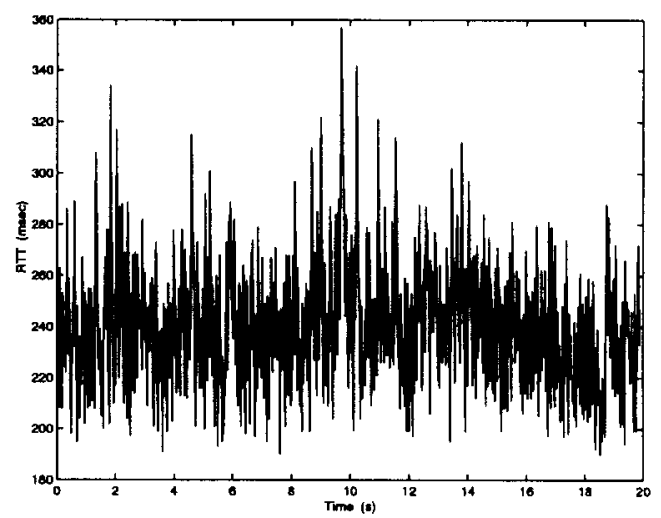

Fig. 5. Time delay from the slave to the master used for the simulation.

needs more control action, easy to cause the input chattering. From this fact, it can be concluded that the regular-form sliding controller is not suitable to be applied for the bilateral teleoperation system with varying time delay, which is shown in later simulations.

\section{Modified Sliding-Mode Controller}

To keep the system performance independently of the presence of varying time delay, we propose a modified sliding-mode controller for the slave as follows:

$$
\begin{aligned}
u_{s}(t)= & B_{s} v_{s}(t)+f_{e}(t)-\frac{k_{p} M_{s}}{M}\left\{B v_{m d}(t)-f_{h d}(t)\right. \\
& \left.+k_{f} f_{e}\left(t-T_{1}-T_{2}\right)+K x_{m d}(t)\right\} \\
& -M_{s} \lambda \dot{\tilde{x}}_{d}(t)-K_{\text {gain }} \cdot \operatorname{sat}\left(\frac{s_{d}}{\Phi}\right)
\end{aligned}
$$

Note that this control law differs from eq. (14) just in the term of $f_{e}\left(t-T_{1}-T_{2}\right)$, substituted with $f_{e}(t)$ in eq. (14). When there is no time delay, this control law is equal to the regular sliding-mode controller, eq. (14). 
With the modified control law, eq. (15) and (16) are changed into:

$$
\begin{gathered}
\dot{s}_{d}(t)+\frac{K_{\text {gain }}}{M_{s}} \cdot \operatorname{sat}\left(\frac{s_{d}}{\Phi}\right)=0 \\
K_{\text {gain }} \geq M_{s} \eta
\end{gathered}
$$

In the above equation, the boundary of $K_{\text {gain }}$ is not the function of time delay, so we can neglect the timedelay effect when select $K_{\text {gain }}$ of the slave controller. If there is no slave model error, $K_{\text {gain }}$, which satisfy eq. (19), guarantees that the behavior of the controlled slave system will satisfy the sliding condition. The overall system block diagram including the master, the slave, and the proposed controllers is shown in Fig. 3.

\section{Simulation}

Performances of the regular and modified slidingmode controller are compared in simulations of a 1DOF bilateral teleoperation system. It is assumed that there is no plant model uncertainty in order to investigate only the time delay effect on the controlled system performance. In this simulation, the plant parameters are set to $M_{m}=0.1[\mathrm{~kg}], B_{m}=0.5[\mathrm{Ns} / \mathrm{m}]$ for the master device and $M_{s}=5[\mathrm{~kg}], B_{s}=1.5[\mathrm{Ns} / \mathrm{m}]$ for the slave.

The slave manipulator has initially position offset with the master position by $0.05[\mathrm{~m}]$. For the impedance controller, the parameters of the desired impedance model are selected as $M=2[\mathrm{~kg}], B=1$ $[\mathrm{Ns} / \mathrm{m}]$, and $K=10[\mathrm{~N} / \mathrm{m}]$. And the scaling factors, $k_{p}$ and $k_{f}$, are set to 1 . Finally, the external environment is modeled as a spring system with $K_{e}=1000$ $[N / m]$.

In order to consider the real network situation into the simulation, the round-trip time (RTT) of a specific network site is measured with the ping-command and these data are used as the varying time delay for the simulation. The time delays from the master to the slave and the slave to the master used for the simulation are shown in Fig. 4 and Fig. 5. These RTTs are about 250 [msec] on the average and change randomly according to the time. Though these values are roundtrip times, they are used as a one-way time delay in the simulation.

\section{A. Simulation Results with Regular Sliding-Mode Con- troller}

For the performance comparison, at first, the gains of the sliding-mode controller such as $K_{\text {gain }}, \lambda$, and $\Phi$ are tuned for the output to show a desirable performance. Then, the varying time delay is applied to the communication block.

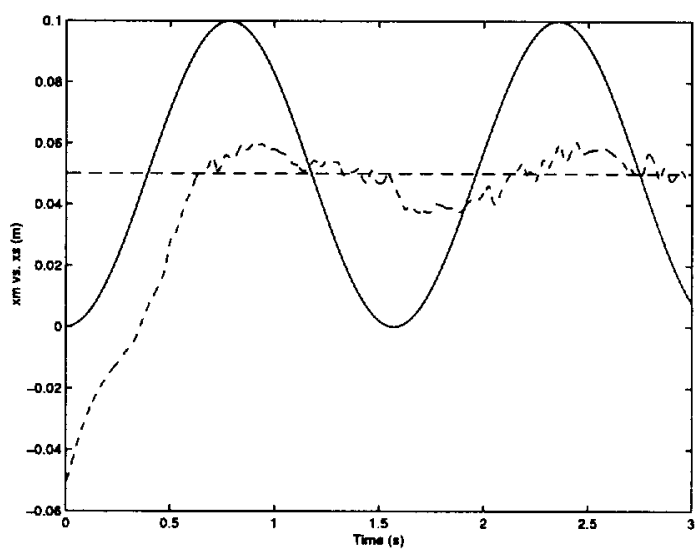

Fig. 6. Position responses of the master and the slave with the regular sliding-mode controller (solid: master, dotted: slave).

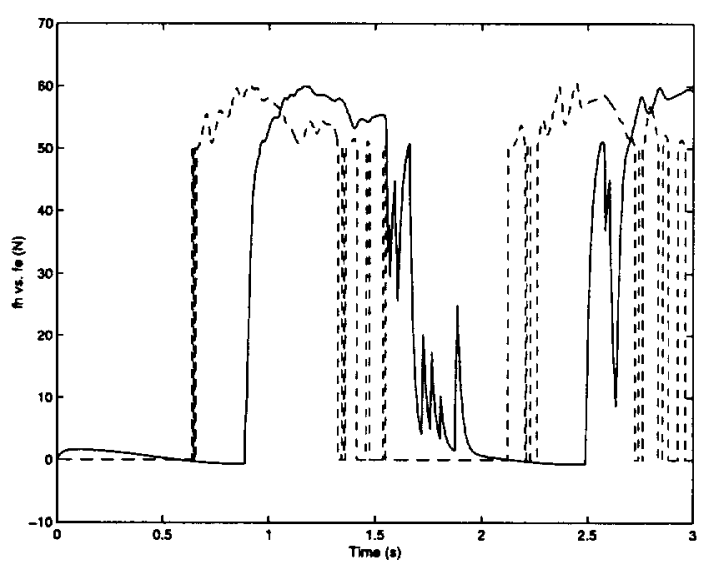

Fig. 7. Force responses of the master and the slave with the regular sliding-mode controller (solid: master, dotted: slave).

Figures 6 and 7 show the position and force responses of the master and the slave when the impedance control (eq. (13)) is used for the master and the regular sliding controller (eq. (14)) for the slave. In Fig. 6, the dotted line is the relative position of the external wall with respect to the master position. The simulation results show that when the slave contacts with the wall, the external force increases suddenly and the nonlinear gain, selected for the case of no time delay is too small to fulfill the sliding condition. Consequently, the varying time delay degrades easily the controller performance.

From this result, it is shown that, with the fixed $K_{\text {gain }}$, the controlled slave may not satisfy the sliding condition and may be unstable according to the time delay variation. In fact, for this simulation, if we set $K_{\text {gain }}$ to be large enough to guarantee the sliding 


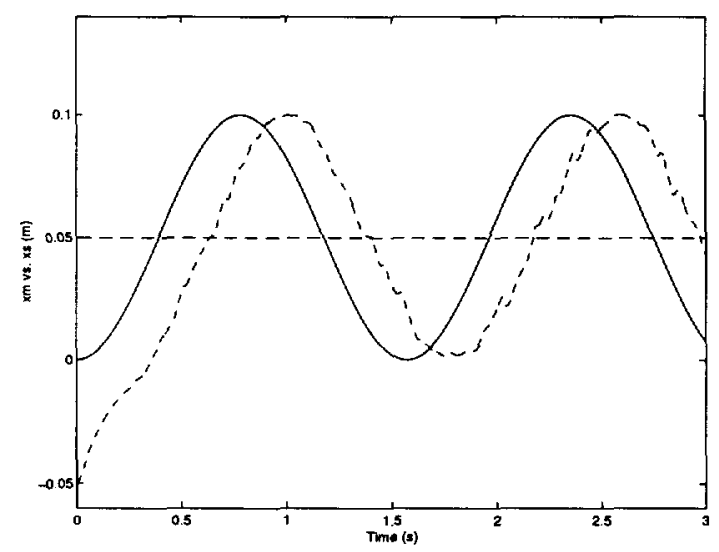

Fig. 8. Position responses of the master and the slave with the proposed sliding-mode controller (solid: master, dotted: slave).

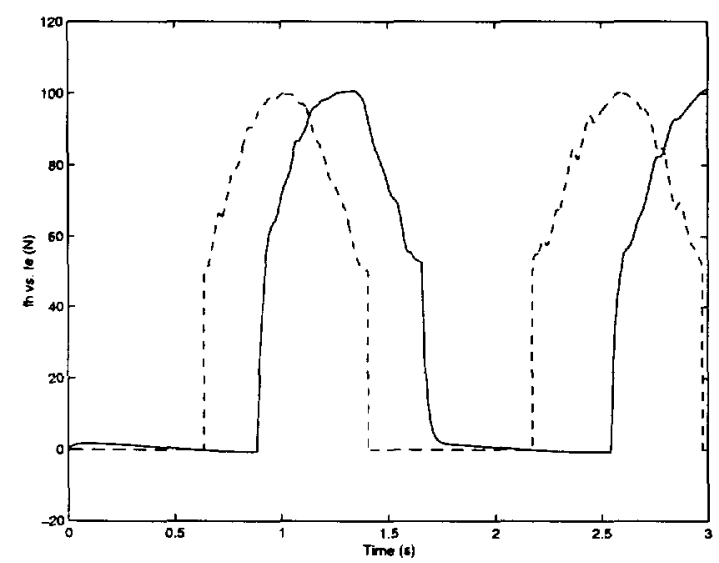

Fig. 9. Force responses of the master and the slave with the proposed sliding-mode controller (solid: master, dotted: slave).

condition, the output of the controlled system shows a satisfactory behavior. It is, however, not so easy to predict trends of time delay and the maximum external force of the slave in a real implementation.

\section{B. Simulation Results with Modified Sliding-Mode Controller}

In this simulatoin, the modified sliding-mode controller (eq. (17)) is used as the slave controller instead of the regular sliding-mode controller. Simulation results are shown in Fig. 8 and Fig. 9.

As shown in eq. (19), in this case, the boundary of the nonlinear gain, $K_{\text {gain }}$ is neither the function of the time delay nor that of the external force. In Fig. 8, the slave position is delayed about 250 [msec] and oscillates slightly due to the time delay variation, but the slave tracks well the delayed master position. And Fig. 9 shows that the external force imposed on the slave is properly reflected to the operator.

\section{Conclusions}

A modified sliding-mode controller, which makes the selection of the nonlinear gain, $K_{\text {gain }}$ be independent of the time delay variation, is suggested. In the regularform sliding controller, in order to guarantee the sliding condition of the slave response, the maximum RTT and the order of the external force must be measured in advance. But, with the proposed controller, the time delay can be neglected in tuning the controller gains and the amount of the time delay need not be measured either. In simulations, it is shown that the proposed sliding-mode controller is superior to the regular-form controller especially when there is severely varying time delay in the communication channel.

\section{REFERENCES}

[1] Robert J. Anderson and Mark W. Spong, "Bilateral Control of Teleoperators with Time Delay," IEEE Transactions on Automatic Control, Vol. 34, pp. 494-501, 1989.

[2] Roberto Oboe and Paolo Fiorini, "A Design and Control Environment for Internet-Based Telerobotics," International Journal of Robotics Research, Vol. 17, pp. 433-449, 1998.

[3] Alessandro Eusebi and Claudio Melchiorri, "Force Reflecting Telemanipulators with Time-Delay: Stability Analysis and Control Design," IEEE Transactions on Robotics and Automation, Vol. 14, pp. 635-640, 1998.

[4] Kevin Brady and Tzyh-Jong Tarn, "Internet-Based Remote Teleoperation," Proceedings of IEEE Int. Conf. on Robotics and Automation, pp. 65-70, 1998.

[5] Akihito Sano, Hideo Fujimoto and Masayuki Tanaka, "Gain-Scheduled Compensation for Time Delay of Bilateral Teleoperation System," Proceedings of IEEE Int. Conf. on Robotics and Automation, pp. 1916-1923, 1998.

[6] Jun Kikuchi, Koji Takeo and Kazuhiro Kosuge, "Teleoperation System via Computer Network for Dynamic Environment," Proceedings of IEEE Int. Conf. on Robotics and Automation, pp. 3534-3539, 1998.

[7] Kazuhiro Kosuge and Hideyuki Murayama, "Bilateral Feedback Control of Telemanipulator via Computer Network in Discrete Time Domain," Proceedings of IEEE Int. Conf. on Robotics and Automation, pp. 2219-2224, 1997.

[8] Günter Niemeyer and Jean-Jacques E. Slotine, "Using Wave Variables for System Analysis and Robot Control," Proceedings of IEEE Int. Conf. on Robotics and Automation, pp. 1619-1625, 1997a.

[9] Günter Niemeyer and Jean-Jacques E. Slotine, "Designing Force Reflecting Tleoperators with Large Time Delays to Appear as Virtual Tools," Proceedings of IEEE Int. Conf. on Robotics and Automation, pp. 2212-2218, 1997b.

[10] Günter Niemeyer and Jean-Jacques E. Slotine, "Towards Force-Reflecting Teleoperation Over the Internet," Proceedings of IEEE Int. Conf. on Robotics and Automation, pp. 1909-1915, 1998.

[11] Pietro Buttolo, Petter Braathen and Blake Hannaford, "Sliding Control of Force Reflecting Teleoperation: Preliminary Studies," PRESENCE, Vol. 3, pp. 158-172, 1994.

[12] Thomas B. Sheridan, Telerobotics, Automation, and $\mathrm{Hu}$ man Supervisory Control, The MIT Press, Cambridge, MA, 1992.

[13] Jean-Jacques E. Slotine and Weiping Li, Applied Nonlinear Control, Prentice-Hall, Inc., 1991. 